\title{
One Type of Bio-Phase Microscopy Imaging Method Based on a Con Beam with Double Dove Prisms
}

\author{
Jingrong Liao ${ }^{1, ~ a ~, ~ J i n g y e ~} \mathrm{Liu}^{2, \mathrm{~b}}$ and Yuanyuan $\mathrm{Xu}^{2, \mathrm{c} *}$ \\ ${ }^{1}$ School of Automotive and Traffic Engineering in Jiangsu University \\ ${ }^{2}$ Faculty of Science in Jiangsu University \\ ajsdxjing@sina.cn, 'lhjsdx@sina.com, cyuanyuanlark126.com \\ *The corresponding author
}

\begin{abstract}
Keywords: Optical interference; Biological cells; Phase imaging; On-axis and off-axis; Double dove prisms
\end{abstract}

\begin{abstract}
Optical interference is an important technique in precision measurement and microscopy imaging. Especially, phase imaging technology of the biological cell has attracted increasing attention. In this paper, considering the high stability characteristics of the coaxial interference technique, one interference imaging system that can do both coaxial and off-axis phase imaging is constructed by using a couple of Dove prisms and a beam splitter without other lens, all the system structure based on a con beam that is simple and can image the phase of a cell after placing a post amplification. It can be obtained by some phase recovery methods for the thickness distribution of a cell, so that, the shape of a homogeneous cell can be shown. Simulation and experimental results have verified the feasibility and correctness of the system. It is one kind of cell morphology analysis technology with simple and practical.
\end{abstract}

\section{Introduction}

Phase imaging is an important technique widely used to the optical measurement, microscopy imaging and so on. Up to now, the phase microscopy imaging technique has been attracted more and more attention, because it is a kind of no contrast agent and without any violation of the light microscopy. In essence, it makes full use of the inherent refractive index of the sample to improve the imaging contrast. Therefore, phase distribution analysis of a transparent body, such as a biological cell, has a very important research value. Especially for a cell with different structures in the intracellular distribution, corresponding to different refractive index (RI) distribution, so RI is an important biochemical parameter, which can be reflected the detail characteristics of the cell structure and change.

Over the decades, quantitative phase microscopy imaging technique(QPI) has been developed and become one kind of important technology in the optical microscopic fields. However, most of imaging techniques are based on the interference principle of two optical waves, since they have the advantages of high detection sensitivity, fast detection rate and high detection accuracy. Now, different kinds of interference imaging systems are produced, such as: Mach-Zehnder (MZ) interferometer [3], phase contrast microscopy interferometer [4], Nomarshi differential interferometer [5], Michelson interferometer [6,7], Linnik interferometer [8,9] and Mirau interferometer [10]. In addition, according to the recording mode, interferometric phase microscopy techniques are mainly classified as two categorites: on-axis and off-axis. Among which, the on-axis technique has the adcantages of high stability and broadband. In general, it is required to combine the phase-shifting technique to retrieval phase .In 1997, the group of Yamaguchi [11] first introduced the phase-shift method to the on-axis digital holography and got an experimental verification. Then, many other phase-shift digital holographic phase microscopy techniques have been successively reported [12-15], such as Awatsuji and etc from 2004 to 2006, respectively, put forward parallel quasi four steps [12] and three phase shift holographic technology [13] to realized 3D images of a object. In 2012, Samsheerali [15] reported a coaxial phase-shift digital holographic technology with the help of a diffraction grating phase, which realized the high stability of the 
quantitative phase imaging. In recent years, other QPI based on the coaxial interference microscopy [16-24] have also been put forward, such as the Popescu team [17] successively put forward Fourier Phase Microscopy (FPM) and space optical interference microscopy (SLIM) [20-23] and the Wax team put forward parallel two-steps phase-shift Microscopy [24], and so on. The research of these techniques have greatly enriched coaxial interference method, make the technology more and more high, but its structure and operation is becoming more and more complicated.

In order to simplify the optical structure of the interference, in this paper, a easy optical structure of a interference imaging system is designed based on the con beam. The system is composed by a BS prism and a double Dove prism(DP). It is placed in one light arm of the Michelson interference. Based on the splitting of BS and combining of DP, we can get a microscopy interference pattern of a small phase body, the coaxial and off-axis interference can be achieved with this system. Simultanously, the phase imaging of the sample can be gotten with the appropriate phase retrieval method.

\section{Phase Microscopy Imaging System}

Michelson interference technique is one of the traditional and mature interference methods. In this work, we use it to perform the quantitative phase imaging. Its structure is depicted in Figure 1. A He-Ne laser is used as the illumination source. To ensure the quality of the beam, it is subsequently collimated from AF(absorptive filter) L1 L2(lens) PH(pin hole). Transform the light path from horizontal to vertical by through a BS(beam spliter). Then, the beam is reflected to two Mirrors (M1and M2) passing through a beam splitter (BS). Among which, the sample is placed on the Mirror 2, thus the reflected light in this illumination area can be regarded as the object beam and that in the illumination area of the Mirror 1 can be regarded as the reference beam. These two parts of beams after BS transmitted enter into double Dove prisms (DP), this two parts are combined into one part by transmission and reflecting of the DP and than goes through a microscope(MO), the interferogram is recorded by a sensor (CMOS). It is worth noting that this experimental setup can carry out several kinds of interferences with different recording modes. For example, in on-axis interference, the mirror M1 is parallel to the mirror M2. In general, the combination of phase-shifting technique is required to retrieve the phase of the sample quantitatively in this case. In our setup, we can use the wave plate $\mathrm{P}$ placed in the reference arm to produce multi-step phase shift. Fig. 2(a) shows the experimental setup in on-axis case, in which, IO and IR present object and reference beams respectively, I presents the interfernce beam. In off-axis interference, the tilt angle between the object and reference beams is not equal to zero. Therefore, it is only needed to adjust the angle of the reflected beam from the mirror M1 in our setup, as shown in Fig. 2(b), in which, a small angle $\theta$ is introduced by tilting the mirror M1, so the angle between IO and IR after transmitting the DP component is $2 \theta$. Thus, our experimental setup can be applied to any an interferometry by adjusting the tilt angle between the mirror M1 and the mirror M2.

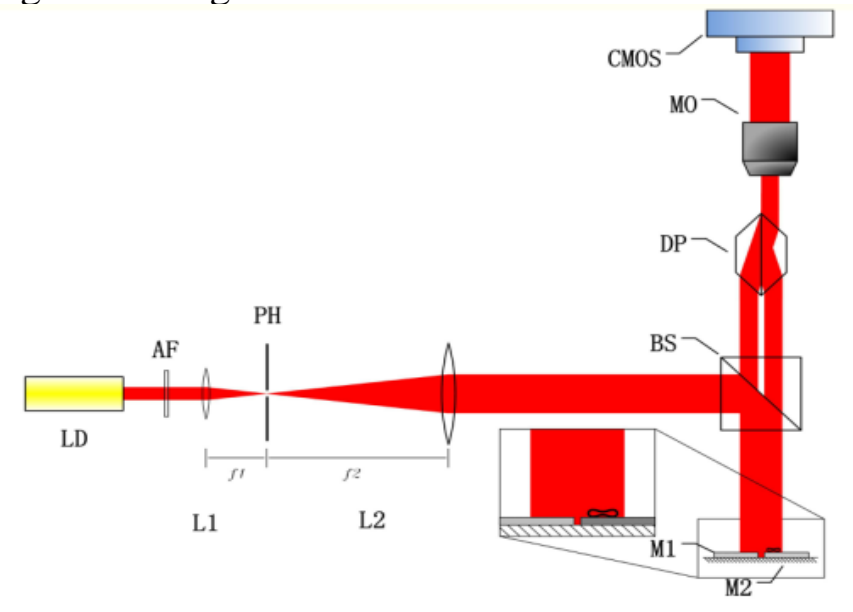

Figure 1. The experimental setup 


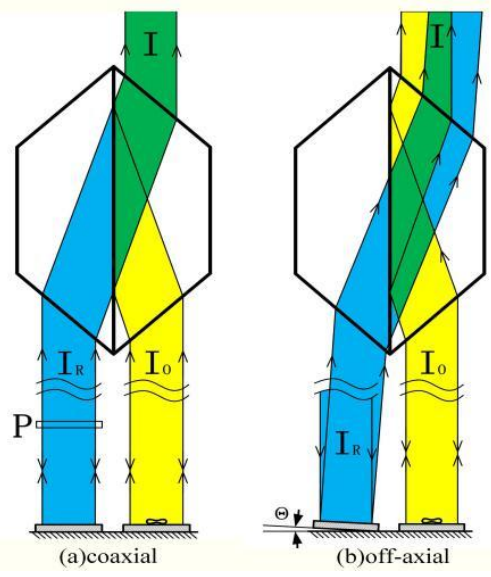

Figure 2. (a) on-axis structure, (b) off-axis structure

\section{Phase Retrieval Methods}

Since the characteristics of interference fringes in on-axis and off-axis interferences are different, the corresponding phase retrieval theories are also different. Here, we will introduce their theories in these two cases.

In on-axis interferometry, the phase-shifting technique is often combined to retrieval the phase information. We assume that two interference waves are plane waves, and the complex amplitudes of object and reference waves are $O(x, y)=A_{O} \exp \left[j \varphi_{O}(x, y)\right], R(x, y)=A_{R} \exp \left[j \varphi_{R}(x, y)\right]$, respectively. In which, ${ }^{A_{O}}$ and $\varphi_{O}(x, y)$ are the amplitude and phase of the object wave, respectively, and $A_{R}$ and $\varphi_{R}(x, y)$ are the corresponding results of the reference wave. According to the interference theory of double beams, the intensity distributions before and after introducing the phase shift can be expressed as

$$
\begin{aligned}
I_{1}(x, y) & =|O(x, y)|^{2}+|R(x, y)|^{2}+2|O(x, y) \| R(x, y)| \cos \left[\varphi_{O}(x, y)-\varphi_{R}(x, y)\right] \\
& =I_{0}(x, y)+\gamma(x, y) \cos [\varphi(x, y)] \\
I_{2}(x, y) & =I_{0}(x, y)+\gamma(x, y) \cos [\varphi(x, y)+\delta] .
\end{aligned}
$$

Here, $I_{0}(x, y)=|O(x, y)|^{2}+|R(x, y)|^{2}$ is the background intensity, $\gamma(x, y)=2|O(x, y)||R(x, y)|$ is the amplitude modulation factor, $\varphi(x, y)=\varphi_{O}(x, y)-\varphi_{R}(x, y)$ is the relative phase difference between the object and reference waves, $\delta$ is the phase shift. To simplify the calculation, $\varphi_{R}(x, y)$ is often assumed as zero, so the relative phase difference is equivalent to the phase of the sample, namely, $\varphi(x, y)=\varphi_{O}(x, y)$. If the phase shift $\delta$ is fixed as a special value of $\pi$, Eq. (2) can be simplified as

$$
I_{2}(x, y)=I_{0}(x, y)-\gamma(x, y) \cos [\varphi(x, y)] .
$$

In this case, the sum of two phase-shifted intensities can be calculated as

$$
I_{1}+I_{2}=2 I_{0}=2\left(|O|^{2}+|R|^{2}\right) \text {. }
$$

Obviously, the background term can be obtained only from two special interference patterns. Thus, it is an alternative method to eliminate the undesired background intensity. In addition, the difference between these two interferograms can be calculated as

$$
I_{1}-I_{2}=2 \gamma(x, y) \cos [\varphi(x, y)]=4|O \| R| \cos [\varphi(x, y)] \text {. }
$$


If the intensity of the reference wave, $I_{R}=|R|^{2}$, is known, the phase can be solved as the following expression

$$
\varphi(x, y)=\arccos \frac{\sqrt{2}\left(I_{1}-I_{2}\right)}{4 \sqrt{\left(I_{1}+I_{2}-2 I_{R}\right) I_{R}}} .
$$

In addition, if we can adjust that the intensity of the object wave is same as that of reference wave, the phase can be solved as

$$
\varphi(x, y)=\arccos \left(\frac{I_{1}-I_{2}}{I_{1}+I_{2}}\right) .
$$

As can be seen from Eqs.(6) and (7), we can get the phase distribution of the sample only from two phase-shifted interferograms and the reference wave. It is need to noted that the phase of a sample measured in our experimental setup is twice as the phase solved with Eq.(6) or Eq.(7), because the double optical paths of a sample by reflecting on the Mirror 2, that does not change the distribution of phase and thickness distribution of the sample. In order to describe convenience, we also call the phase in Eq.(6) is the phase of the sample.

In the off-axis interferometry, owing to that the object and reference waves are not parallel, the linear phase factor is introduced, and the phase can be retrieved only by single an interferogram. The interference intensity can be given by

$$
I(x, y)=I_{0}(x, y)+\gamma(x, y) \cos [\varphi(x, y)+k x] .
$$

where $I_{0}(x, y), \gamma(x, y)$ and $\varphi(x, y)$ are the same as those in on-axis case. $k$ is the spatial frequency of the fringes. In this case, the background intensity can be eliminated by a high pass filter or an average algorithm. Consequently, we can obtain the interference term, which is given by

$$
I^{(0)}=\gamma \cos [\varphi(x, y)+k x] .
$$

Then, we apply the fast derivative algorithm to process the interference term. Considering the real case, we have the following approximation:

$$
\frac{\partial I_{0}}{\partial x} \approx 0, \frac{\partial \gamma}{\partial x} \approx 0, \frac{\partial^{2} \varphi}{\partial x^{2}} \approx 0
$$

This approximation of the phase is easily satisfied, even for some complex structured phase objects, and it is verified in Ref. [25]. Thus, the 1st and 2nd order derivatives of Eq. (9) can be approximated as

$$
\begin{aligned}
& I^{(1)}=-\gamma \sin [\varphi(x, y)+k x]\left(\frac{\partial \varphi}{\partial x}+k\right) . \\
& I^{(2)}=-\gamma \cos [\varphi(x, y)+k x]\left(\frac{\partial \varphi}{\partial x}+k\right)^{2} .
\end{aligned}
$$

Then, according to Eqs. (9), (11) and (12), the phase of a sample can be expressed as 


$$
\varphi(x, y)=\left\{\begin{array}{l}
\tan ^{-1}\left\{\left[-\frac{\left[I^{(1)}\right]^{2}}{I^{(2)} \cdot I^{(0)}}\right]^{1 / 2}\right\}-k x \quad\left(I^{(1)} \cdot I^{(2)} \geq 0\right) \\
\tan ^{-1}\left\{-\left[-\frac{\left[I^{(1)}\right]^{2}}{I^{(2)} \cdot I^{(0)}}\right]^{1 / 2}\right\}-k x \quad\left(I^{(1)} \cdot I^{(2)}<0\right)
\end{array}\right.
$$

\section{Simulation and Experimental Validation}

In order to demonstrate the ability of phase imaging system designed, we take off-axis interferometry as an example, and carry out the simulation and experiment of a tiny ball.

In our simulationm, the radius and the refractive index of the ball are set as 2 um and 1.59. Other parameters are as follows: the refractive index of the environment liquid is 1.57 , the amplitudes of the object and reference waves are unit, the wavelength of the light source is $632.8 \mathrm{~nm}$, the spatial frequency $k$ is $1.0683 \mathrm{rad} / \mathrm{pixel}$, and the size of the interference pattern recorded by a CCD is $512 \times 512$ pixels. According to these associated parameters and the phase retrieval theory in off-axis interferometry mentioned in Section 2, we can perform the phase imaging quantitatively. Fig. 3(a) is the interferogram.Fig. 3(b) is the interference term after high-pass filtering. Figs. 3(c) and 3(d) are the 1st and 2nd order derivatives of Fig. 3(a). According to Eq.(13), we can obtain the phase distribution of the ball, as shown in Fig. 3(e). Since the ball is homogeneous, there is a linear relationship between the axial thickness and phase distribution. To be specific, assuming that the light propagates along z-axis, this relationship can be expressed as

$$
\varphi(x, y)=\frac{2 \pi}{\lambda} \cdot \Delta n \cdot H=\frac{2 \pi}{\lambda} \cdot \Delta n \cdot\left[2 \sqrt{R^{2}-x^{2}-y^{2}}\right] .
$$

In which, $H$ is the thickness along with the optical axis, and $\Delta n$ is the refractive index difference between the ball and the environment liquid. So the thickness information can be calculated from the measured phase directly, and the result is presented in Fig.3(f). Via the calculation, the maximum thickness of the ball is $4.0072 \mu \mathrm{m}$, and the corresponding error is $0.0072 \mu \mathrm{m}$, which is very small compared with the theoretical value. It shows that this phase retrieval method is feasible and high accuracy.
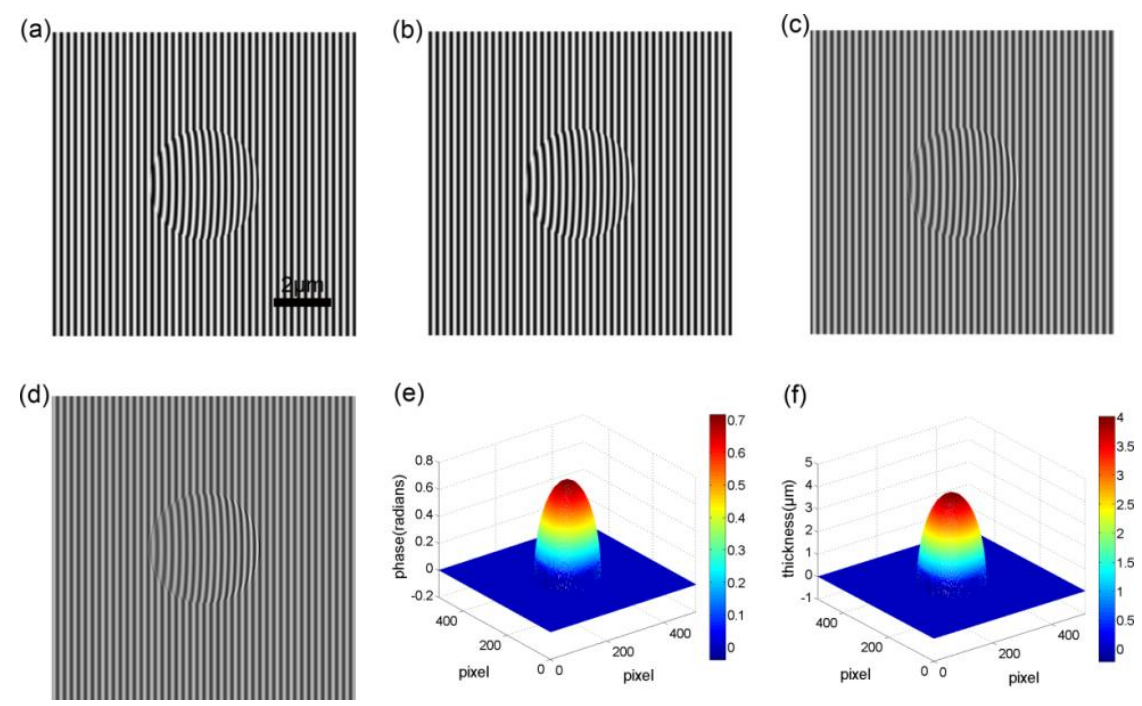

Figure 3. Simulation experiment. (a) interfeogram, (b) interference item, (c) the first order derivative of (a), (d) the second orderderivativeof (a), (e) the reconstruction phase, (f) the reconstruction thickness 
In the experiment, we carry out the phase imaging of a $\mathrm{SiO} 2$ ball with the diameter of $1.5 \mathrm{~mm}$ in off-axis interfeometry. The experimental setup is shown in Fig.2(b). Parameters owned by some main components are as follows: A He-Na laser (LD180) with the wavelength of $632.8 \mathrm{~nm}$ is selected as the light source, the beam diameter after extending is $6 \mathrm{~mm}$, magnification of the object lens (Navitar P Can) is $20 \mathrm{x}$, the resolution of a CMOS (Infinity 1) is $1024 \times 1024$ pixels, a Double Dove prisms ( $450 \mathrm{~nm} \sim 650 \mathrm{~nm}$ ) is used to combine the reference and object waves, a BS mirror ( $450 \mathrm{~nm} \sim 650 \mathrm{~nm}$ ) is used to split the light wave, and the parameters of the reflecting mirror is $R_{\text {avg }}>99.7 \%$ and $520 \mathrm{~nm} \sim 647 \mathrm{~nm}$.

The experimental result is shown in Fig. 4 based on the principle of Fig.1. Fig. 4(a) shows the micrograph of a SiO2 ball, Figs. 4(b) and 4(c) show the interferograms before and after placing the ball, Fig. 4(d) shows the phase distribution. Comparing the experimental results and the simulation results, it is shown that there are a basically agreement between the sample's phase distribution in experiment and in the simulation results.
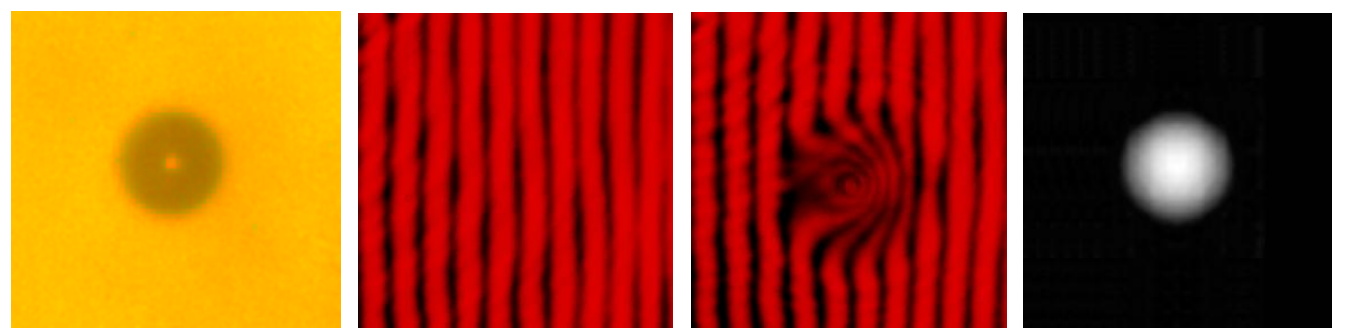

Figure 4. Experimental results of a $\mathrm{SiO} 2$ ball . (a) the micrograph, (b) the interferogram without the ball, (c) the interferogram with the ball, (d) the reconstructed phase

\section{Conclusion}

In the paper, a system applied to a interference phase imaging, is designed. This system has the advantages of high stability and broadband, because the on-axis imaging method in the Michelson interferometer can be realized. Moreover, the system reduces the spherical aberration due to the less using of lens. It is easy to get the coaxial or off-axis interference patterns only by putting the wave plate in reference beam or change the enter angle, the phase and thickness distribution can be gotten with the appropriate phase retrieval method. The feasibility of the designed system is verified by a simulation calculation and an experiment. It is one kind of simple phase microscopy imaging system, which can be used in QPI.

\section{Acknowledgments}

This work was supported by National Natural Science Foundation of China under Grant Nos. 11604127 and 11374130.

\section{Reference}

[1] C.L. Curl, C.J. Bellair, P.J. Harris,B.E. Allman, A. Roberts, Quantitative phase microscopy: A new tool for investigating the structure and function of unstained live cells $[\mathrm{J}]$. Clin. Exp. Pharmacol. P., 2004, 31(12):896-901.

[2] Y. Wang, D. Wang, Y. Yang, J.Zhao, L. Ouyang. Application and Analysis in the Biomedicine Field Using Digital Holographic Technology [J]. Chinese Journal of Lasers , 2014, 41(2): 0209002(1-13).

[3] C. Fang-Yen, S. Oh, Y. Park, W. Choi, S. Song, Imaging voltage-dependent cell motions with heterodyne Mach-Zehnder phase microscopy [J]. Opt. Lett., 2007, 32(11): 1572-1574. 
[4] P. Gao, B.L. Yao, I. Harder, et al. Phase-shifting Zernike phase contrast microscopy for quantitative phase measurement [J]. Opt. Lett., 2011, 36(21): 4305-4307.

[5] X. Cui, M. Lew, C. Yang, Quantitative differential interference contrast microscopy based on structured-aperture interference [J]. Appl. Phys. Lett., 2008, 93(9):091113(1-3).

[6] B. Kemper, A. Vollmer, C.E. Rommel, J. Schnekenburger, B.G. Von, Simplified approach for quantitative digital holographic phase contrast imaging of living cells [J]. J. Biomed. Opt., 2011, 16(2): 026014.

[7] R.L. Guo, B.L. Yao, P. Gao, et al. Parallel on-axis phase-shifting holographic phase microscopy based on reflective point-diffraction interferometer with long-term stability [J]. Appl. Opt., 2013, 52(13):3484-3489.

[8] A. Safrani, I. Abdulhalim, Real-time phase shift interference microscopy [J]. Opt. Lett., 2014, 39(17):5220-5223.

[9] R.L. Guo, B.L. Yao, J.W. Min, et al. LED-based digital holographic microscopy with slightly off-axis interferometry [J]. J. Opt., 2014, 16(12):125408.

[10] M. Leon-Rodrigue, R. Rodriguez-Vera, J.A. Rayas, et al. Digital holographic microscopy through a Mirau interferometric objective [J]. Opt. Lasers Eng., 2013, 51(3): 240-245.

[11] Yamaguchi, T. Zhang, Phase-shifting digital holography [J]. Opt. Lett., 1997, 22(16):1268-1270.

[12] Y. Awatsuji, M. Sasada, T. Kubota, Parallel quasi-phase-shifting digital holography [J]. Appl. Phys. Lett., 2004, 85(6):1069-1071.

[13] A. Yasuhiro, F. Atsushi, K. Toshihiro, et al. Parallel three-step phase-shifting digital holography [J]. Appl. Opt., 2006, 45(13):2995-3002

[14]B.G. Hao, M.G. Shan, Z. Zhong, et al. Common-path interferometer with four simultaneous phase-shifted interferograms using ronchi grating and cube beamsplitter [J]. Opt. Lasers Eng., 2013, 51(11): 1278-1282.

[15]P.T. Samsheerali, B. Das, J. Joseph, Quantitative phase contrast imaging using common-path in-line digital holography [J]. Opt. Commun., 2012, 285(6): 1062-1065.

[16]C. Meneses-Fabian, G. Rodrigue-Zunita, J.F. Vazquez-Sanchez, et al. Common-path phase-shifting interferometer with binary grating [J]. Opt. Common., 2006, 264(1):13-17.

[17] G.Popescu, L.P. Deflores, J.C. Vaughan, et al. Fourier phase microscopy for investigation of biological structures and dynamics [J]. Opt. Lett., 2004, 29(21): 2503-2505.

[18] N. Lue, W. Choi, G. Popescu, et al. Quantitative phase imaging of live cells using fast Fourier phase microscopy [J]. Appl. Optics, 2007, 46(10): 1836-1842.

[19] B.Bhaduri, K. Tangella, G. Popescu, Fourier phase microscopy with white light [J]. Biomed. Opt. Express, 2013, 4(8): 143-1441.

[20]Z. Wang, L. Millet, M. Mir, et al. Spatial light interference microscopy (SLIM) [J]. Opt. Express, 2011, 19(2): 1016-1026.

[21] S. Sridharan, M. Mir, G. Popescu, Simultaneous optical measurements of cell motility and growth [J]. Biomed. Opt. Express, 2011, 2(10): 2815-2820.

[22]Z. Wang, L. Millet, V. Chan, et al. Label-free intracellular transport measured by spatial light interference microscopy [J]. J. Biomed. Opt., 2011, 16(2), 026019(1-9). 
[23] H.Majeed, G. Popescu, Breast cancer diagnosis using spatial light interference microscopy [J]. J. Biomed. Opt., 2015, 20(11):111210(1-6).

[24] N.T. Shaked, T.M. Newpher, M.D. Ehlers, et al. Parallel on-axis holographic phase microscopy of biological cells and unicellular microorganism dynamics [J]. Appl. Opt., 2010, 49(15): 2872-2878.

[25] Xu Yuanyuan, COL 12(Suppl.), S11001(2014) CHINESE OPTICS LETTERS April 30, 2014,S11001-1-4 